\title{
UCP2 silencing aggravates mitochondrial dysfunction in astrocytes under septic conditions
}

\author{
WANWAN PENG ${ }^{1,2 *}$, JINDA HUANG $^{3 *}$, YIJUN ZHENG ${ }^{4}$, YUE DING $^{1}$, SITAO $^{2}{ }^{5}$, \\ JUNLIANG ZHANG $^{6}$, JUANJUAN LYU ${ }^{7,8}$ and QIYI ZENG ${ }^{1,2}$
}

\begin{abstract}
${ }^{1}$ Department of Pediatrics, Zhujiang Hospital, Southern Medical University, Guangzhou, Guangdong 510280;
${ }^{2}$ Guangzhou Key Laboratory of Inflammatory and Immune Diseases, Guangzhou, Guangdong 510515;

${ }^{3}$ Pediatric Intensive Care Unit, Guangzhou Women and Children's Medical Center; ${ }^{4}$ Department of Neonatology,

Guangzhou Women and Children's Medical Center, Guangzhou, Guangdong 510623; ${ }^{5}$ Department of Pediatrics, The Sixth

Affiliated Hospital of Sun Yat-sen University, Guangzhou, Guangdong 510655; ${ }^{6}$ Department of Neonatology, Nanfang

Hospital, Southern Medical University, Guangzhou, Guangdong 510515; ${ }^{7}$ Department of Pediatrics, West China Second

University Hospital, Sichuan University; ${ }^{8}$ Key Laboratory of Birth Defects and Related Diseases of Women and Children, Ministry of Education, West China Second University Hospital, Sichuan University, Chengdu, Sichuan 610041, P.R. China
\end{abstract}

Received February 9, 2019; Accepted July 18, 2019

DOI: $10.3892 / \mathrm{mmr} .2019 .10721$

\begin{abstract}
Uncoupling protein 2 (UCP2) plays a positive role in sepsis. However, the role of UCP2 in experimental sepsis in astrocytes remains unknown. The present study was designed to determine whether UCP2 has a protective effect in an experimental sepsis model in astrocytes asnd to clarify the mechanisms responsible for its neuroprotective effects after sepsis. An experimental astrocyte model mimicking sepsis-induced brain injury was established using lipopolysaccharide (LPS) and interferon (IFN)- $\gamma$. Additionally, UCP2 knockdown in astrocytes was achieved by adenovirus transfection. Tumor necrosis factor (TNF)- $\alpha$ and interleukin (IL)-1 $\beta$ activity, mitochondrial membrane potential (MMP) and reactive oxygen species (ROS), and adenosine triphosphate (ATP) levels were assessed. The mitochondrial ultrastructure was evaluated, and the expression of UCP2 was determined by western blotting. LPS with IFN- $\gamma$ co-stimulation increased the mRNA and protein expression levels of UCP2 in astrocytes, damaged the mitochondrial structure, and accelerated
\end{abstract}

Correspondence to: Dr Juanjuan Lyu, Department of Pediatrics, West China Second University Hospital, Sichuan University, Section 3, 20 Renmin South Road Chengdu, Sichuan 610041, P.R. China

E-mail: juanjuanlyu@163.com

Dr Qiyi Zeng, Department of Pediatrics, Zhujiang Hospital, Southern Medical University, 253 Middle Industrial Road, Haizhu, Guangzhou, Guangdong 510280, P.R. China

E-mail: zqy_88@163.com

*Contributed equally

Key words: astrocytes, sepsis-associated encephalopathy, UCP2, mitochondrial dysfunction, reactive oxygen species the release of TNF- $\alpha$ and IL- $1 \beta$, resulting in a decrease in the MMP, and the excessive generation of ROS. Moreover, sepsis also caused a reduction in ATP production. The knockdown of UCP2 exacerbated astrocyte injury and mitochondrial impairment. In conclusion, both the function and morphology of mitochondria were damaged in an experimental model of sepsis in astrocytes, and knockdown of UCP2 using shRNA exacerbated this impairment, suggesting that UCP2 has a positive effect on astrocytes as determined in an experimental sepsis model.

\section{Introduction}

Sepsis is an organ dysfunction caused by an imbalance altered homeostasis in the host due to infection. Its high morbidity and mortality, resulting in the increased use of medical resources, is the main cause of death in pediatric intensive care units (PICUs) (1). Sepsis-associated encephalopathy (SAE) is a diffuse brain dysfunction caused by sepsis, that can occur during its early stages, even before the onset of clinical symptoms. The pathogenesis of SAE involves multiple factors and is complex $(2,3)$. Many studies of both septic patients and animal models have concluded that mitochondrial disruption appears to be related to the progression and outcome of sepsis $(4,5)$. A previous study (6) demonstrated that elements of mitochondrial disorder are conducive to SAE, including increases in reactive oxygen species (ROS), and mitochondrial biogenesis, generation of cellular ATP and reduction in the mitochondrial membrane potential (MMP or $\Delta \Psi \mathrm{m}$ ). Astrocytes account for $90 \%$ of the total brain cells and play an important role in the pathophysiology of the central nervous system (CNS) (7). Therefore, we explored the changes in mitochondrial function in astrocytes under septic conditions.

According to published data, uncoupling protein 2 (UCP2) plays a vital role under physiological and pathological conditions (8). This main role of UCP2 is to regulate the mitochondrial 
inner and outer membrane proton gradients and establish a bridge between ATP and ROS generation (4). Increased UCP2 expression has been observed during pathological states such as atherosclerotic plaques (9) cerebral ischemia (10), experimental autoimmune encephalomyelitis (EAE) (11), and lipopolysaccharide (LPS)-induced cardiomyocyte injury (12). Some pathophysiological conditions can cause irritation that can lead to increased UCP2 expression, resulting in a neuroprotective process. UCP2 alleviates ROS generation to protect cells from oxidative stress-induced damage. UCP2 has been implicated in intracellular calcium regulation, neuronal plasticity, ATP production, apoptosis and synaptic transmission (13).

Inflammatory signaling activation is an important mechanism leading to increased ROS production and mitochondrial dysfunction (14). In experimental sepsis, after co-stimulation with LPS and interferon (IFN) $-\gamma$, a potent inflammatory state and significant mitochondrial damage can be observed $(6,15)$. UCP2 is considered an important neuroprotective element in many inflammatory and degenerative states of the CNS (16). For more than five years, our group has been dedicated to studying the role of UCP2 and mitochondrial dysfunction in the pathophysiology of sepsis. Previous studies by our team have demonstrated that the total expression of UCP 2 is increased in brain tissue (17), which is consistent with other findings (11). However, the exact function of UCP2 in astrocytes under experimental sepsis conditions remains unknown. It is also unknown whether UCP2 has a positive effect on astrocytes under experimental sepsis conditions by maintaining the MMP and the production of ROS. We speculated that UCP2 may regulate the MMP and mitochondrial function in experimental sepsis models. In this study, synthesis of ROS and ATP, the MMP level, and other related factors were investigated following the knockdown of UCP2 by adenoviral transduction in primary astrocytes that were used as an experimental sepsis model.

\section{Materials and methods}

Chemicals and reagents. Dulbecco's modified Eagle's medium (DMEM), fetal bovine serum (FBS) and trypsin were purchased from Gibco; Thermo Fisher Scientific, Inc. (Waltham, MA, USA). The following polyclonal primary antibodies were used in this study: Anti-UCP2 (1:1,000, 89326; Cell Signaling Technology, Inc.), anti-GFAP (1:200, 80788; Cell Signaling Technology, Inc.) and anti- $\beta$-actin $(1: 5,000$, AP0060; Bioworld Technology, Inc.).

Cell culture. Neonatal rat cerebral cortical astrocytes were prepared using enzymatic dissociation and culture from 1-day-old newborn Sprague-Dawley rat brains from animals obtained from the Laboratory Animal Center of Southern Medical University (Guangzhou, China) according to a protocol described previously (6). This study was approval by the Nanfang Hospital Ethics Committee (NFYY-2017-39). The animals used in the study were provided by Southern Medical University Animal Experiment Center [SCXK(Guangdong)2016-0041], and passed the experimental animal quality inspection in Guangdong Province (no. 44002100020527).
Establishment of cell sepsis model. Astrocytes isolated from neonatal rat cerebral codex were purified and cultured, and then randomly allocated into 4 groups: i) Control group, ii) LPS+IFN- $\gamma+6 \mathrm{~h}$ group, iii) LPS+IFN- $\gamma+12 \mathrm{~h}$ group and iv) LPS+IFN- $\gamma+24$ h group. The cells were stimulated with LPS (150 ng/ml from Escherichia coli O111:B4; Sigma-Aldrich; Merck KGaA) and IFN- $\gamma(200 \mathrm{U} / \mathrm{ml}$; Thermo Fisher Scientific, Inc.)

Adenoviral transduction. The recombinant adenoviruses used to knockdown UCP2 (AD-UCP2) were designed and synthesized by Gene Chem Co. (Shanghai, China). The cells were transfected by viruses according to the manufacturer's instructions and stimulated with LPS $(150 \mathrm{ng} / \mathrm{ml}$ from Escherichia coli O111:B4; Sigma-Aldrich; Merck KGaA) and IFN- $\gamma(200 \mathrm{U} / \mathrm{ml}$; Thermo Fisher Scientific, Inc.) at $24 \mathrm{~h}$ after adenoviral transduction and for 48-72 $\mathrm{h}$ before the subsequent detection.

Determination of the mRNA levels of tumor necrosis factor (TNF)- $\alpha$, interleukin (IL)-1 $\beta$ and UCP2. Total RNA was extracted from the cultured cells using TRIzol reagent (Takara, Dalian, China). Reverse transcription was conducted with a One-Step RNA-PCR Kit (Takara) according to the manufacturer's instructions. $\beta$-actin was used as an internal control for the real-time PCR amplification. The sequences of the primers for real-time PCR analysis were as follows: $\beta$-actin forward, 5'-AACACACGAGACGCTGAAGT-3' and reverse primer, 5'-TCCAGTGAGTTCCGAAAGCC-3'; UCP2 forward, 5'-GCTGGTGGTGGTCGGAGAT-3' and reverse primer, 5'-TGAAGTGGCAAGGGAGGT-3'; TNF- $\alpha$ forward, 5'-AACACACGAGACGCTGAAGT-3' and reverse primer, 5'-TCCAGTGAGTTCCGAAAGCC-3'; IL-1 $\beta$ forward, 5'-CCT TGTCGAGAATGGGCAGT-3' and reverse primer, 5'-TTC TGTCGACAATGCTGCCT-3'. Quantitative real-time PCR was performed on a Roche Lightcycler 480 Real-Time PCR System using SYBR Premix Ex Taq ${ }^{\mathrm{TM}}$ (Takara Shuzo Co., Kyoto, Japan). After adding the corresponding primers and cDNA to the master mix, the PCR thermal cycle parameters were as follows: $95^{\circ} \mathrm{C}$ for $10 \mathrm{~min}, 40$ cycles at $60^{\circ} \mathrm{C}$ for $60 \mathrm{sec}$ and $95^{\circ} \mathrm{C}$ for $15 \mathrm{sec}$, and a melting curve from $60^{\circ} \mathrm{C}$ to $95^{\circ} \mathrm{C}$, which was performed to ensure amplification of a single product. The $\beta$-actin gene was used as an internal control to normalize for differences in the amount of total RNA in each sample. Calculations were based on the $2^{-\Delta \Delta C q}$ method using the equation $\mathrm{R}$ (ratio) $=2^{-\Delta \Delta \mathrm{Cq}}$ and normalized to $\beta$-actin expression in each sample (18).

Enzyme-linked immunosorbent assay (ELISA) for the detection of $I L-1 \beta$ and TNF- $\alpha$. ELISA kits (Cusabio, Wuhan, China) were used to determine the protein levels of TNF- $\alpha$ and IL-1 $\beta$ in the cultured supernatant. All procedures were performed according to the manufacturer's instructions.

Transmission electron microscopy (TEM). After groups of astrocytes were treated, they were collected and fixed in $2 \%$ glutaraldehyde at $4^{\circ} \mathrm{C}$ overnight. The cells were dehydrated, embedded in epoxy resin (Piano) and polymerized after fixation. The cells were then stained with uranyl acetate and lead citrate and imaged using a Hitachi transmission electron microscope (magnification x20,000; H-7650; Hitachi, Tokyo, Japan). 

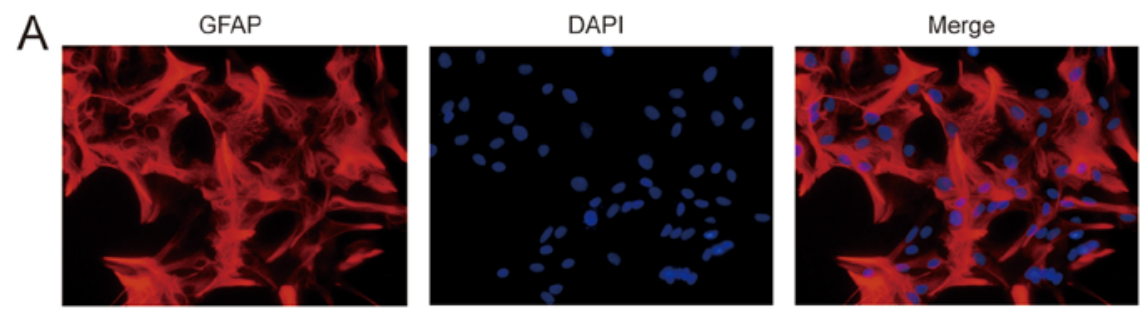

B

UCP2

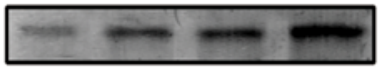

$\beta$-actin
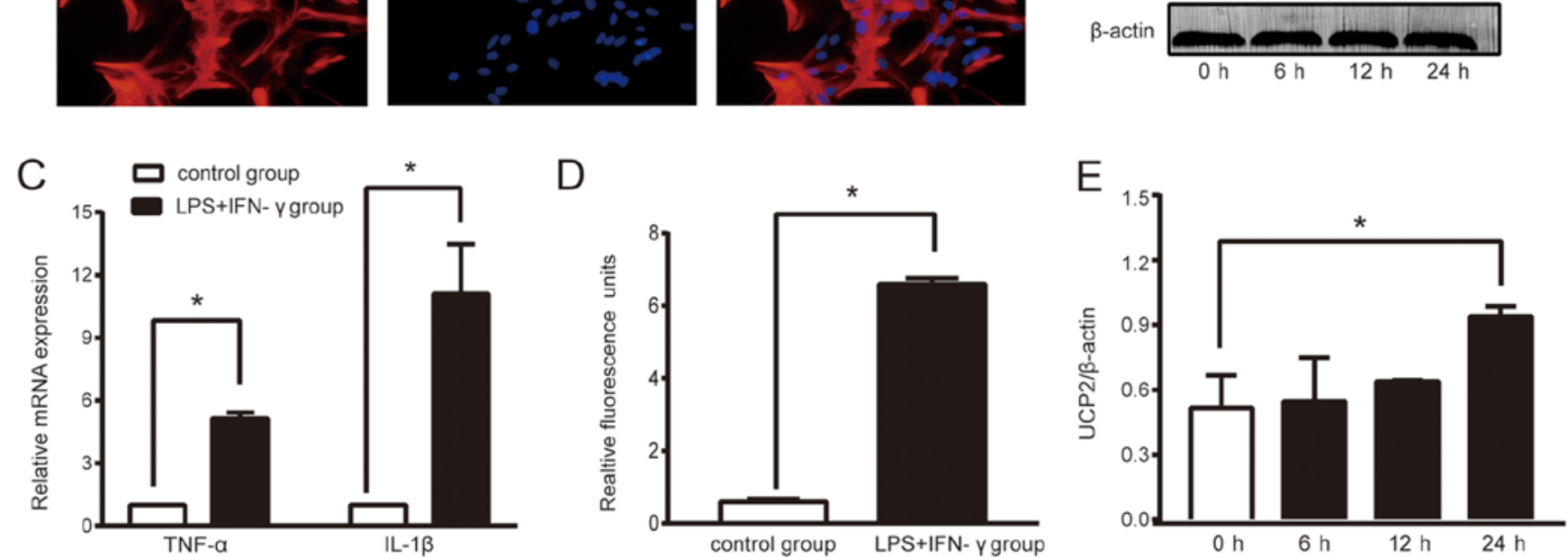

Figure 1. LPS+IFN- $\gamma$ treatment successfully triggers sepsis and increases UCP2 expression in astrocytes. (A) The proportion of astrocytes in primary cultured cells was more than 95\%, which met the requirements of this experiment (original magnification $\mathrm{x} 400$ ). (B) Relative fold change in the protein levels of UCP2. Astrocytes treated with LPS+IFN- $\gamma$ for $0,6,12,24 \mathrm{~h}$ were analyzed by western blot analysis. (C) Expression of TNF- $\alpha$ and IL-1 $\beta$ mRNA in the two cell groups. (D) ROS level. Data are the means $\pm \mathrm{SD}$ ( $\mathrm{n}=3-5$ in each group). (E) Grayscale analysis of relative fold change in the protein levels of UCP2. " $\mathrm{P}<0.05$ vs. the control or $0 \mathrm{~h}$ group. UCP2, uncoupling protein 2 ; LPS, lipopolysaccharide; IFN- $\gamma$, interferon- $\gamma$; TNF- $\alpha$, tumor necrosis factor $\alpha$; IL-1 $\beta$, interleukin-1 $\beta$; ROS, reactive oxygen species.

Measurement of intracellular ROS levels. Intracellular ROS generation was assessed by 2,7-dichlorodihydrofluorescein diacetate (DCFH-DA; Sigma-Aldrich; Merck KGaA). Astrocytes $\left(7.0 \times 10^{5}\right.$ cells/well) were treated with culture medium containing $10 \mu \mathrm{M}$ DCFH-DA for $30 \mathrm{~min}$ at $37^{\circ} \mathrm{C}$. After incubation, the cells were assessed using a fluorescence microscope (magnification $\mathrm{x} 400$; Olympus Corporation), and the intensity of fluorescence was quantified by flow cytometry (BD Biosciences).

Measurement of the MMP. The MMP was assessed in astrocytes using a JC-1 kit (Beyotime, Jiangsu, China). After treatment, the cells $\left(1.5 \times 10^{5}\right.$ cells/well $)$ were incubated with JC-1 staining solution at $37^{\circ} \mathrm{C}$ for $25 \mathrm{~min}$ and then washed twice with JC-1 staining buffer. MMP was detected by flow cytometry (BD Biosciences), and cells were imaged using a confocal microscope (magnification x400; Olympus Corporation).

Measurement of intracellular ATP levels. The ATP levels in astrocytes were assessed using an ATP assay kit (Beyotime, Jiangsu, China) according to the manufacturer's instructions. Data were normalized to the protein concentration of the cell.

Immunofluorescence staining. Astrocytes $\left(1.5 \times 10^{5}\right.$ cells/well) were fixed in $4 \%$ paraformaldehyde for $15 \mathrm{~min}$ and permeabilized in $0.2 \%$ Triton X-100 for 15 min. Then, the cells were blocked with $5 \%$ bovine serum albumin in TBS for $1 \mathrm{~h}$ and incubated with primary antibodies (1:200) at $4^{\circ} \mathrm{C}$ overnight. Next, the cells were incubated with the appropriate secondary antibody $(1: 5,000$, ab150079) for $60 \mathrm{~min}$ at room temperature. Finally, the nuclei were stained with DAPI for $15 \mathrm{~min}$. After staining the cells were imaged using a fluorescence microscope (magnification x400; Olympus Corporation).
Western blot analysis. Total protein was extracted using a Bradford Protein Assay Kit (KeyGEN, Jiangsu, China). Each protein sample $(30 \mu \mathrm{g})$ was separated on an SDS-polyacrylamide gel (10\%) and then transferred onto a PVDF filter membrane (Millipore). The membranes were blocked in 5\% BSA for $60 \mathrm{~min}$ at room temperature and incubated with the specified primary antibodies $(1: 1,000)$ at $4^{\circ} \mathrm{C}$ overnight. Subsequently the membranes were incubated with HRP-conjugated secondary antibodies (1:10,000, BS13278; Bioworld Technology). Densitometry was performed by using ImageJ software 1.8.0 (National Institutes of Health, Bethesda, MD, USA).

Statistical analysis. SPSS 25.0 (IBM Corp., Armonk, NY, USA) was used to analyze the experimental data, and values are expressed as the mean \pm SD. Statistical analyses were performed using either one-way analysis of variance (ANOVA) followed by post hoc pairwise comparison (LSD) tests for analysis, or Student's t-tests and a value of $\mathrm{P}<0.05$ was considered statistically significant.

\section{Results}

Cell identification. To ensure the accuracy of the experiments, the purity of the primary cells cultured were determined during the 4th week of culture. We labeled the astrocytes with specific protein, glial fibrillary acidic protein (GFAP), by immunochemical staining. According to the observed results (Fig. 1A), the proportion of astrocytes in the primary cultured cells was $>95 \%$, which met the requirements of this experiment.

Biomarkers of sepsis. Sepsis is caused by infection. During sepsis, proinflammatory cytokine secretion and ROS 
A

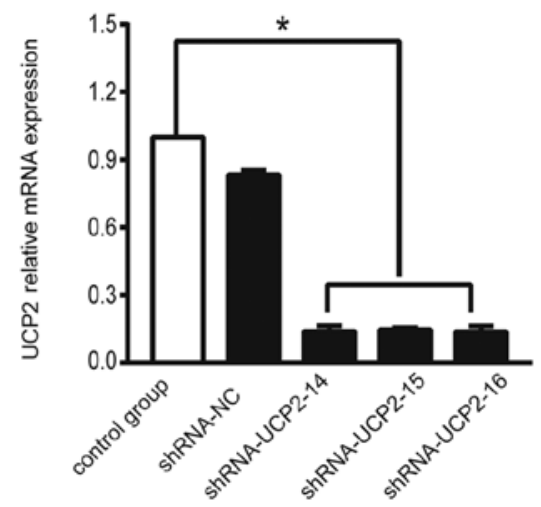

$\mathrm{D}$

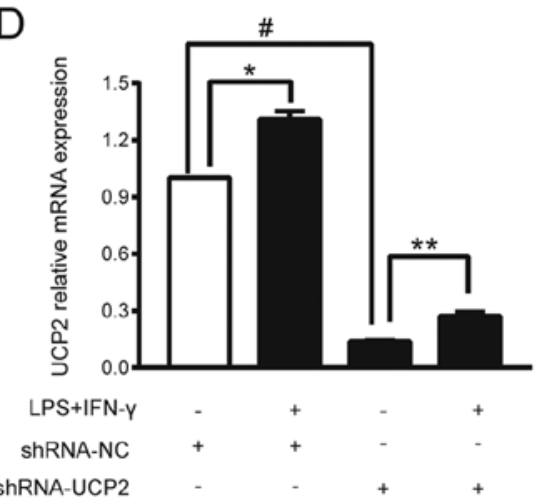

B

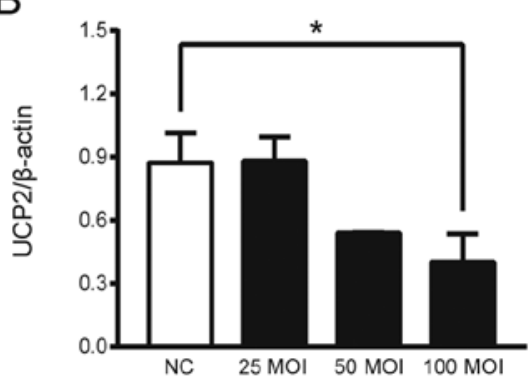

C

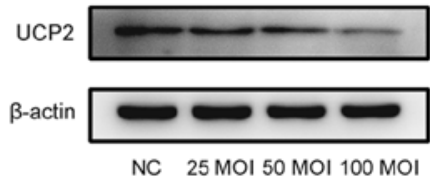

E

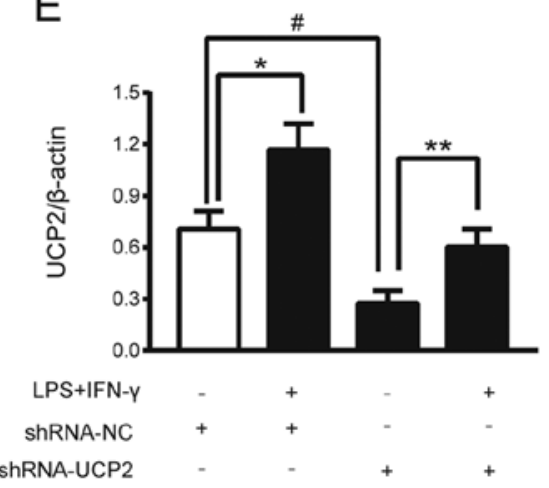

$\mathrm{F}$

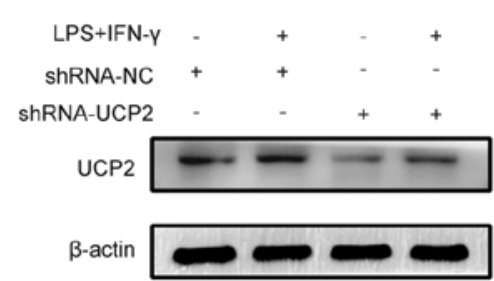

Figure 2. Successful adenoviral transduction and knockdown efficiency of UCP2. (A) Knockdown efficiency of shRNA-UCP2 in the astrocytes. The mRNA levels of UCP2 were determined by RT-qPCR with normalization to $\beta$-actin. ${ }^{*} \mathrm{P}<0.05$ vs. the control group. (B and $\mathrm{C}$ ) Immunoblotting showing the expression of UCP2 at different concentrations (MOI) of sh-UCP2. ${ }^{*} \mathrm{P}<0.05$ vs. the shRNA-NC group. (D) Compared to the shRNA-UCP2 group, sepsis caused a 2.08 -fold increase in UCP2 mRNA as noted in the LPS+IFN- $\gamma+\operatorname{shRNA-UCP2}$ group. ${ }^{*} \mathrm{P}<0.05$ vs. shRNA-NC group; ${ }^{* *} \mathrm{P}<0.05$ vs. shRNA-UCP2 group; ${ }^{\#} \mathrm{P}<0.01$ vs. shRNA-NC group. (E and F) Compared to the shRNA-UCP2 group, sepsis caused a 2.22 -fold increase in UCP2 protein expression as noted in the LPS+IFN- $\gamma+$ shRNA-UCP2 group. ${ }^{*} \mathrm{P}<0.05$ vs. shRNA-NC group; ${ }^{* *} \mathrm{P}<0.05$ vs. shRNA-UCP2 group; ${ }^{*} \mathrm{P}<0.01$ vs. shRNA-NC group. UCP2, uncoupling protein 2 ; LPS, lipopolysaccharide; IFN- $\gamma$, interferon- $\gamma$; MOI, multiplicity of infection.

generation are increased, which damages the brain. After stimulation of astrocytes with LPS $(150 \mathrm{ng} / \mathrm{ml})$ and IFN- $\gamma$ $(200 \mathrm{U} / \mathrm{ml})$, the mRNA levels of TNF- $\alpha$ and IL-1 $\beta$ were increased in the supernatant of cultured cells compared to the control group $(\mathrm{P}<0.05$; Fig. 1C). The ROS level was significantly higher in the LPS+IFN- $\gamma$ group than that noted in the control group $(\mathrm{P}<0.05$, Fig. 1D). Thus, compared to the control group, treatment with LPS+IFN- $\gamma$ induced a 6.59-fold increase in the ROS level, a 5.13-fold increase in the TNF- $\alpha$ mRNA level and a 11.09-fold increase the IL-1 $\beta$ mRNA level. These results showed that an astrocyte sepsis model had been successfully created.

Sepsis increases the expression levels of UCP2 in astrocytes. To assess the expression of UCP2 in an experimental sepsis model in astrocytes, we performed a time course study of UCP2. The western blot results showed that UCP2 protein levels increased following sepsis induction and reached a peak expression level at $24 \mathrm{~h}$. Compared to the control group, sepsis caused a 1.82-fold increase in UCP2 protein expression $(\mathrm{P}<0.05$; Fig. 1B and E).

Transfection efficiency of UCP2. UCP2 expression in astrocytes was successfully knocked down by transfection with adenoviral-shUCP2, which was performed according to the manufacturer's instructions. The mRNA levels of UCP2, which represent the knockdown efficiency of UCP2, were measured by RT-qPCR at $72 \mathrm{~h}$ after transfection. After transfection with shRNA-UCP2-14, the mRNA level of UCP2 was reduced by $88 \%$, and transfection with shRNA-UCP2-15 and shRNA-UCP2-16 resulted in reductions of $85 \%$ compared to the control group ( $\mathrm{P}<0.05$; Fig. $2 \mathrm{~A})$. shRNA-UCP2-14 was thus selected for use in the subsequent experiments. Western blot analysis revealed that an MOI of 25 to 100 for each astrocyte was able to reduce the expression of UCP2, and an MOI of 100 showed a higher inhibition efficiency $(\mathrm{P}<0.05$; Fig. $2 \mathrm{~B}$ and $\mathrm{C})$.

UCP2 $\mathrm{mRNA}$ and protein levels are increased in transfected astrocytes in an experimental sepsis model. To assess the role of UCP2 in the astrocyte sepsis model after UCP2 knockdown, astrocytes were transfected with adenoviral-shUCP2 (shRNA-UCP2) to suppress UCP2 expression. After adenoviral transduction for $48 \mathrm{~h}$, the cells were treated with LPS+IFN- $\gamma$ or DMEM for $2 \mathrm{~h}$. The total RNA and protein levels of UCP2 were analyzed. Compared to the shRNA-NC group, shRNA-UCP2 induced a 0.87 -fold decrease in UCP2 mRNA and a 0.39 -fold decrease in UCP2 protein expression suggesting that UCP2 expression was successfully suppressed $\left({ }^{*} \mathrm{P}<0.01\right.$; Fig. 2D-F). Compared to the shRNA-UCP2 group, sepsis caused a 2.08-fold increase in UCP2 mRNA and a 2.22-fold increase in UCP2 protein expression as noted in the LPS+IFN- $\gamma+$ shRNA-UCP2 group $\left({ }^{* *} \mathrm{P}<0.05\right.$; Fig. 2D-F). 
A

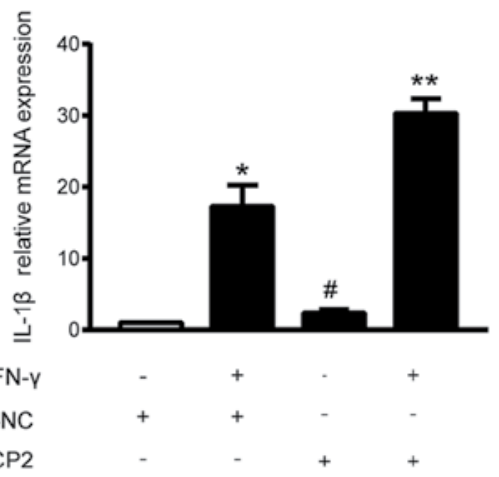

ShRNA-UCP2

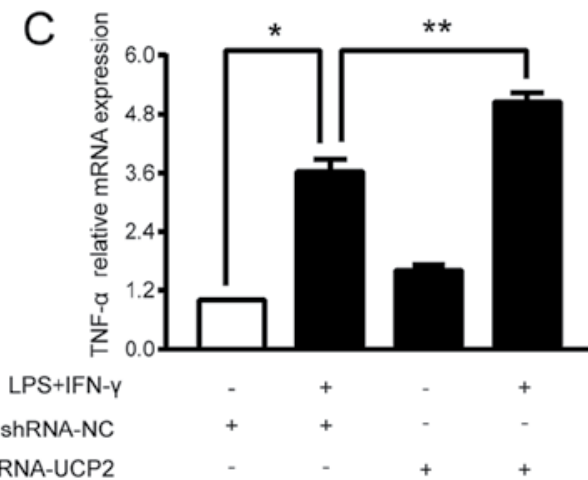

B
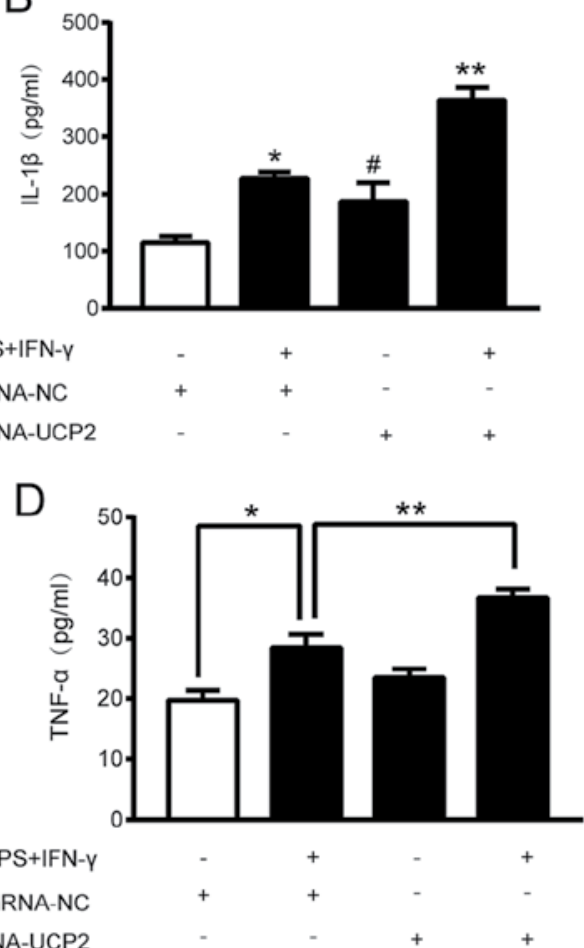

E

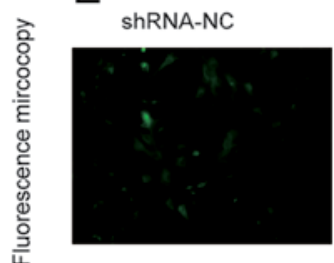

shRNA-NC+LPS+IFN-Y

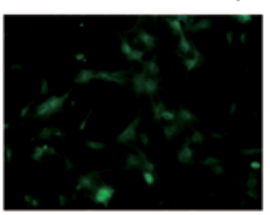

$\mathrm{F}$

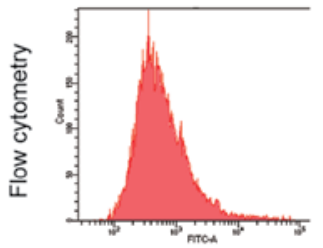

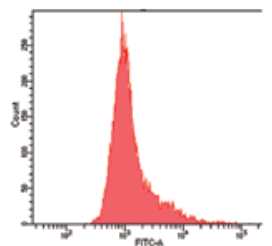

ShRNA-UCP2
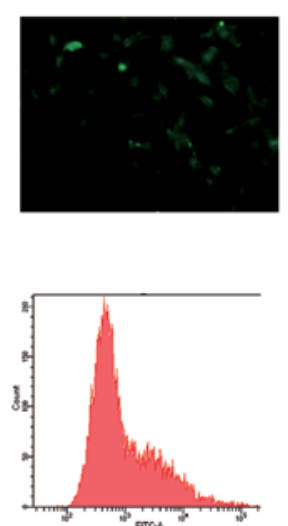

ShRNA-UCP2+LPS+IFN- -
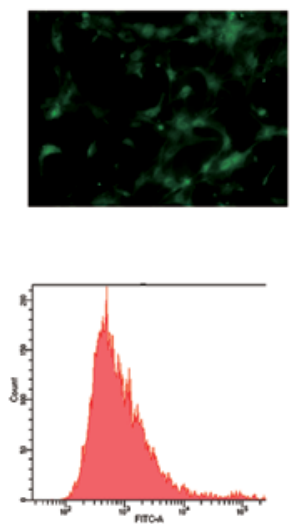

G

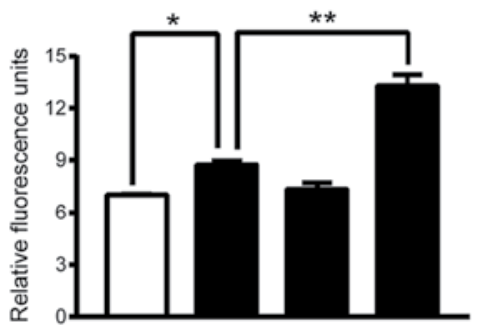

LPS+IFN-Y ShRNA-NC ShRNA-UCP2

Figure 3. Effect of UCP2 knockdown on LPS+IFN- $\gamma$-induced astrocyte damage and oxidative stress. Astrocytes were transduced with shRNA-NC or shRNA-UCP2 adenovirus followed by treatment with or without LPS+IFN- $\gamma$. (A and B) The levels of IL- $1 \beta$ in the astrocytes were assessed by RT-PCR and ELISA kits. (C and D) The levels of TNF- $\alpha$ in the astrocytes were measured by RT-PCR and ELISA kits. "P<0.05 vs. shRNA-NC group; ${ }^{* *} \mathrm{P}<0.05$ vs. LPS+IFN- $\gamma+$ shRNA-NC group. \#P $<0.05$ vs. shRNA-NC group. (E) ROS production was determined by assessing representative immunofluorescence images (original magnification $\mathrm{x} 400$ ). ( $\mathrm{F}$ and $\mathrm{G}$ ) Astrocytes were stained with DCFH-DA followed by flow cytometry (F) and luminometry. (G) The data are presented as the means $\pm \mathrm{SD}(\mathrm{n}=3)$. ${ }^{*} \mathrm{P}<0.05$ vs. shRNA-NC group; ${ }^{* *} \mathrm{P}<0.05$ vs. LPS+IFN- $\gamma+\operatorname{shRNA}$-NC group. UCP2, uncoupling protein 2 ; LPS, lipopolysaccharide; IFN- $\gamma$, interferon- $\gamma$; TNF- $\alpha$, tumor necrosis factor- $\alpha$; IL-1 $\beta$, interleukin- $1 \beta$; ROS, reactive oxygen species.

The results showed that UCP2 expression was enhanced at the mRNA and protein levels after stimulation with LPS+IFN- $\gamma$.

UCP2 knockdown enhances TNF-a and IL-1 $\beta$ levels in an experimental sepsis model in astrocytes. TNF- $\alpha$ and IL-1 $\beta$ are important indicators of sepsis. Treatment with shRNA-UCP2 slightly increased the TNF- $\alpha$ and IL- $1 \beta \mathrm{mRNA}$ and protein expression levels. As shown in Fig. 3A-D, compared to the shRNA-NC group, treatment with shRNA-UCP2 induced a 1.62-fold increase in the TNF- $\alpha$ mRNA level $(\mathrm{P}<0.05)$ resulting in a TNF- $\alpha$ concentration in the culture medium of $23.44 \pm 1.5(\mathrm{pg} / \mathrm{ml})$, and a 3.15-fold increase the IL-1 $\beta$ mRNA level
$(\mathrm{P}<0.05)$ resulting in a IL-1 $\beta$ concentration in the culture medium of $187.46 \pm 32.19(\mathrm{pg} / \mathrm{ml})$. Subsequently, we explored the effects of shRNA-UCP2 on astrocytes under septic conditions. mRNA and supernatant from cells treated with LPS + IFN- $\gamma+$ shRNA-UCP2 had significantly higher IL- $1 \beta$ and TNF- $\alpha$ concentrations and mRNA levels (all $\mathrm{P}<0.05)$ than the LPS+IFN- $\gamma+$ shRNA-NC group. This significant increase indicated that UCP2 knockdown enhances the TNF- $\alpha$ and IL-1 $\beta$ levels in astrocytes in an experimental sepsis model.

UCP2 knockdown exacerbated ROS elevation in astrocytes under septic conditions. To investigate the role of UCP2 

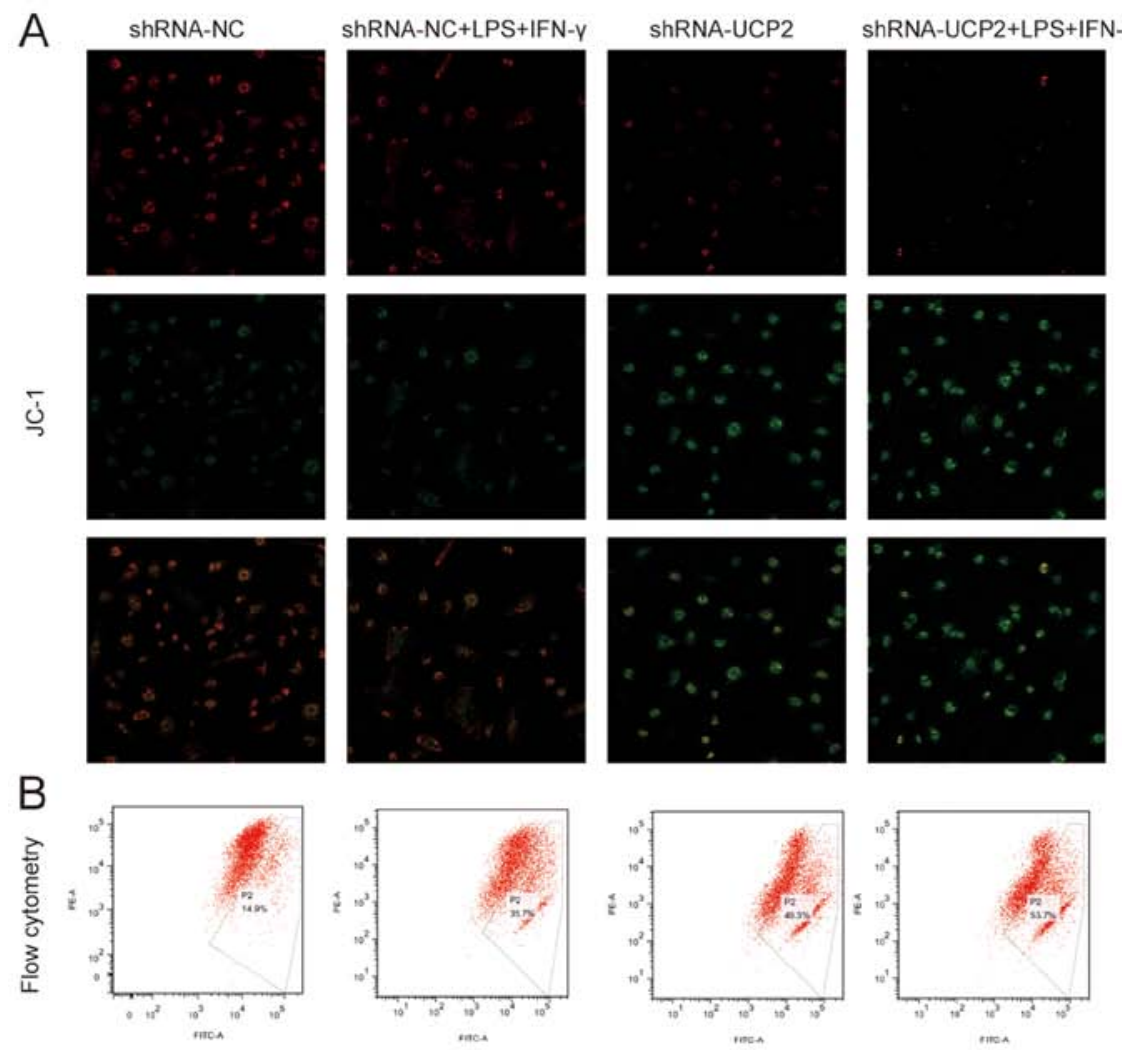

$\operatorname{rnes}$
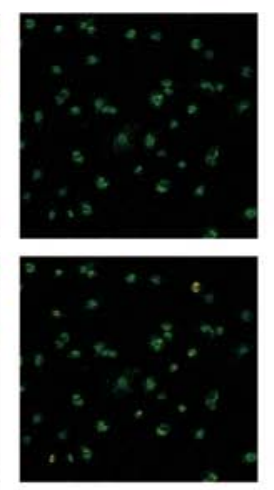

$\mathrm{E}$
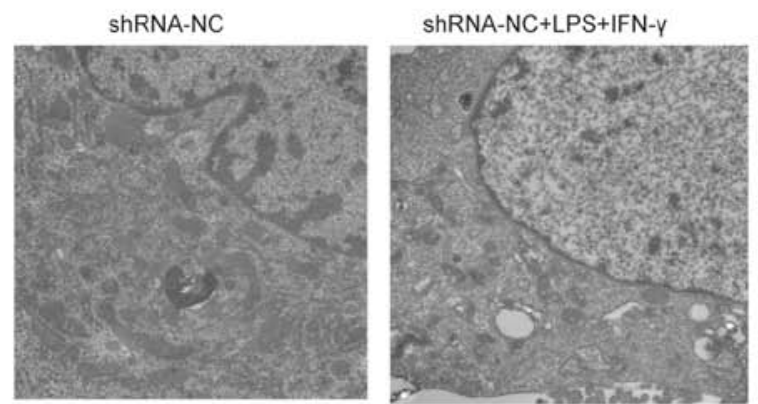
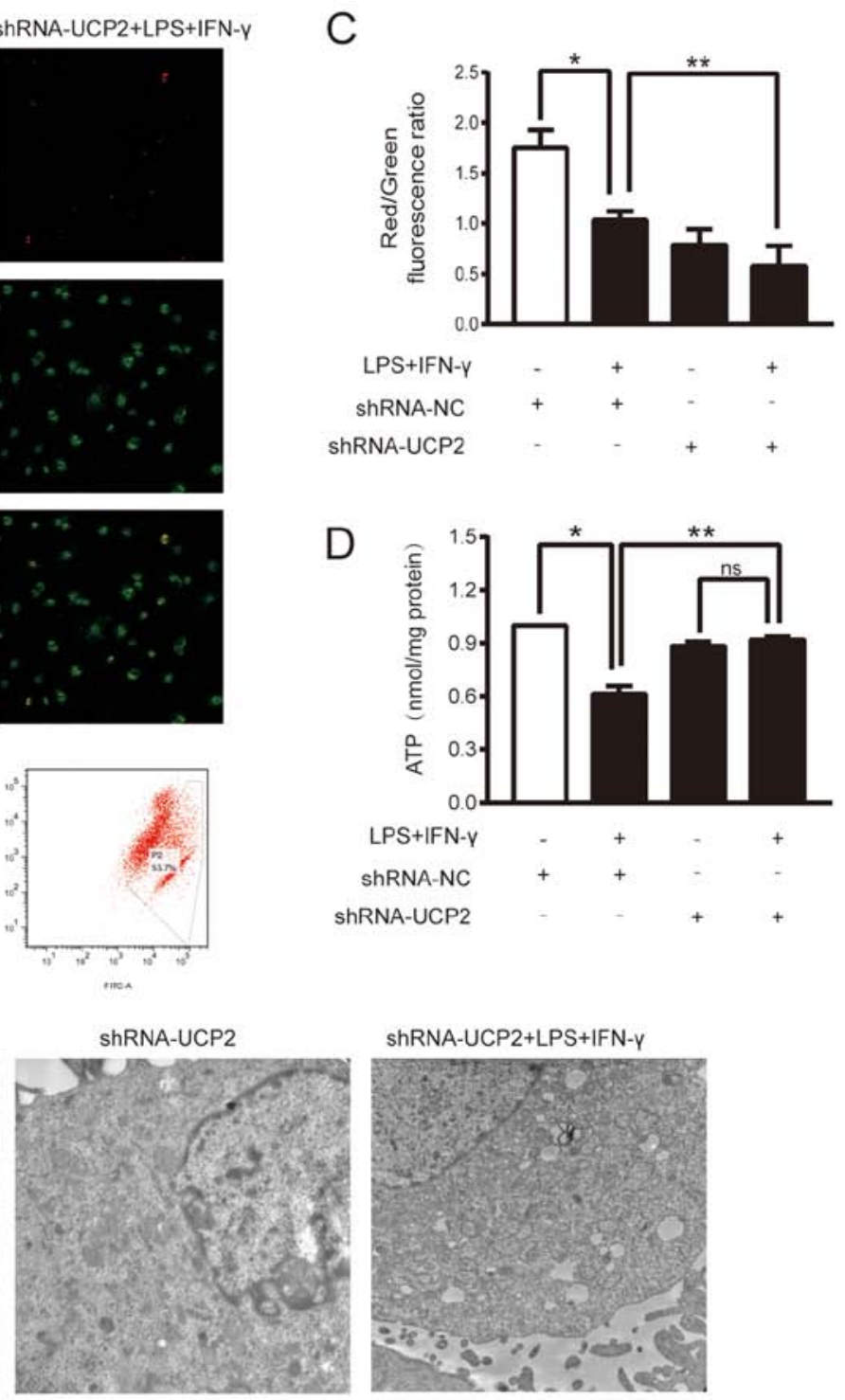

Figure 4. Effect of UCP2 knockdown on mitochondrial function and morphology after LPS+IFN- $\gamma$ treatment. Astrocytes were transduced with Ad-shRNA-NC or Ad-shRNA-UCP2 adenovirus followed by treatment with or without LPS+IFN- $\gamma$. (A-C) Astrocytes were stained with JC-1 to determine the mitochondrial membrane potential $(\Delta \Psi \mathrm{m})$ by confocal microscopy (original magnification, $\mathrm{x} 400)(\mathrm{A})$ and flow cytometry (B and $\mathrm{C}$ ) ${ }^{*} \mathrm{P}<0.05$ vs. shRNA-NC group; ${ }^{* *} \mathrm{P}<0.05$ vs. LPS $+\mathrm{IFN}-\gamma+$ shRNA-NC group. (D) Intracellular ATP levels were determined with an ATP assay kit with a luminometer. ${ }^{*} \mathrm{P}<0.05$ vs. shRNA-NC group; ${ }^{* *} \mathrm{P}<0.05$ vs. LPS+IFN- $\gamma+$ shRNA-NC group; ns, not significant. Data are presented as the means \pm SD (n=3). (E) The mitochondrial ultrastructure was imaged using a transmission electron microscope (original magnification x 20,000). UCP2, uncoupling protein 2 ; LPS, lipopolysaccharide; IFN- $\gamma$, interferon- $\gamma$; ATP, adenosine triphosphate.

in mitochondrial function, the intracellular ROS level of each group was measured by using DCFH-DA probes. Compared to the shRNA-NC group, treatment with LPS and IFN- $\gamma$ induced a 1.24-fold increase in ROS generation in the LPS+IFN- $\gamma+$ shRNA-NC group $(\mathrm{P}<0.05)$. Compared to the LPS+IFN- $\gamma+$ shRNA-NC group, shRNA-UCP2 caused a 1.52-fold-increase in ROS generation in the LPS+IFN- $\gamma+$ shRNA-UCP2 group $(P<0.05$; Fig. 3E-G). The results indicated that UCP2 knockdown significantly increased the ROS generation in astrocytes after treatment with LPS+IFN- $\gamma$ for $24 \mathrm{~h}$.

UCP2 knockdown aggravates mitochondrial dysfunction in an experimental sepsis model in astrocytes. To further evaluate the effects of UCP2 knockdown on LPS+IFN- $\gamma$-induced injury, we analyzed the MMP and the ATP levels in astrocytes after various treatments. The ratio of red to green fluorescence was determined to indicate the MMP value. As shown in Fig. 4A, the red fluorescence intensity in the LPS+IFN- $\gamma+$ shRNA-UCP2 group was weaker than that in the LPS+IFN- $\gamma+$ shRNA-NC group. However, the opposite results were observed for the green fluorescence intensity. To further determine the change in the MMP, the ratio of red fluorescence to green fluorescence as shown by flow cytometry was assessed (Fig. 4B and C). In the shRNA-NC group, the ratio was $1.589 \pm 0.429$, while the LPS+IFN- $\gamma+$ shRNA-NC group showed a lower ratio $(1.033 \pm 0.089 ; \mathrm{P}<0.05$ vs. the shRNA-NC group, $\mathrm{n}=3)$, indicating a decrease in the MMP in the astrocytes under experimental sepsis. Astrocytes treated with LPS+IFN- $\gamma+$ shRNA-UCP2 exhibited a further decline, with a ratio of $0.578 \pm 0.202,(\mathrm{P}<0.05$ vs. LPS + IFN- $\gamma+$ shRNA-NC group, $n=3)$. The results indicated that knockdown of UCP2 exacerbated the decrease in the 
MMP as detected by confocal microscopy and flow cytometry. Cellular ATP detection showed that after $24 \mathrm{~h}$ of exposure to LPS+IFN- $\gamma$, the cellular ATP content was markedly reduced in the shRNA-NC+LPS+IFN- $\gamma$ group compared to the shRNA-NC group and the shRNA-UCP2+LPS+IFN- $\gamma$ group $(\mathrm{P}<0.05$; Fig. 4D). However, compared to that of the shRNA-UCP2 group, the cellular ATP content in the shRNA-UCP2+LPS+IFN- $\gamma$ group was not significantly (ns) different (P>0.05; Fig. 4D).

UCP2 knockdown increases mitochondrial ultrastructure damage in astrocytes under septic conditions. The mitochondrial ultrastructure was examined by TEM (Fig. 4E), which showed that most mitochondria of astrocytes in the shRNA-NC group had little injury, while damage was increased in the shRNA-NC+LPS+IFN- $\gamma$ group, with some swelling and even vacuolization in mitochondria. Compared to the shRNA-NC group, knockdown of UCP2 significantly enhanced mitochondrial swelling damage. Furthermore, after stimulation of UCP2 knockdown in astrocytes with LPS+IFN- $\gamma$, the mitochondrial ultrastructure was most severely damaged; increased mitochondrial vacuolization and rupture of mitochondrial membranes were observed.

\section{Discussion}

Our study findings demonstrated that astrocytes subjected to an in vitro model of sepsis-associated encephalopathy (SAE) induced by lipopolysaccharide (LPS) and interferon (IFN)- $\gamma$ exhibited increased expression of uncoupling protein 2 (UCP2) during the early stage. Adenoviral transduction successfully decreased UCP2 mRNA and protein expression in cells during SAE. In addition, astrocytes with adenoviral transduction of shUCP2 that were stimulated with LPS+IFN- $\gamma$ showed an increase in reactive oxygen species (ROS) production, marked by damage to the mitochondrion, higher UCP2 protein levels, and decreases in the mitochondrial membrane potential (MMP) and cellular adenosine triphosphate (ATP) levels. When astrocytes with UCP2 knocked down were stimulated by LPS+IFN- $\gamma$, we observed an increase in proinflammatory marker expression and damage to the mitochondrial ultrastructure.

Our group has reported the mechanisms of sepsis-induced mitochondrial dysfunction in various organs; these mechanisms are gradually assuming an important role in sepsis research and its potential treatment (6,19-21). To establish the mechanisms underlying the sepsis-induced brain inflammatory process, we have observed numerous cellular and molecular changes that, with time and increased understanding, broaden the list of new molecules that may contribute to the pathological state of the disease. While UCP2-based neuroprotection has been widely reported in many species $(22,23)$ its participation in SAE requires further investigation. Increased expression of UCP2 was observed after LPS+IFN- $\gamma$ was added to the primary astrocytes, reaching a peak at $24 \mathrm{~h}$ after stimulation. Combined stimulation with LPS + IFN $-\gamma$ previously showed greater efficiency in activating astrocytes in vitro than stimulation with LPS alone (6).

The silencing of UCP2 gene expression is a molecular protocol that targets the mRNA of this molecule, limiting its availability by annealing with complementary nucleotide sequences. Lu et al (22) demonstrated that UCP2 deficiency increased intracellular ROS generation and elevated oxidative stress response 1-methyl-4-phenylpyridinium treatment in primary astrocytes. Moreover, Deng et al (14) reported that overexpression of UCP2 in A549 cells inhibited ROS accumulation and apoptosis under hypoxic conditions. The authors assumed that the induction of UCP2 expression in the selected cells could be considered an endogenous protective mechanism that might effectively reduce the stimulation of inflammation. Our observation that knockdown of UCP2 in primary astrocytes made them more susceptible to the mitochondrial dysfunction might be evidence of the neuroprotection elicited by UCP2.

The MMP is formed by the asymmetric distribution of protons and other ions on both sides of the membrane. Stabilization of the MMP is conducive to maintaining the normal physiological function of cells. As previously demonstrated, a decrease in the MMP is closely related to cell damage (24). In the present study, stimulation with LPS+IFN- $\gamma$ led to a decrease in the MMP in an experimental sepsis model in astrocytes, and knockdown of UCP2 markedly aggravated mitochondrial damage (12), which is consistent with our previous study that analyzed LPS-induced sepsis in neonatal rat cardiomyocytes (unpublished data). UCP2 regulates the proton concentration of the mitochondrial inner and outer membranes. Therefore, UCP2 is strongly associated with the MMP, as indicated by the fact that in LPS-induced cardiomyocyte injury, overexpression of UCP2 can markedly change the MMP and alter ROS generation. Many studies have shown an interaction between the MMP and ROS (19,25). UCP2 is considered an important factor in increasing neuronal survival after stroke and brain trauma, and its activation can reduce ROS production and cellular stress (10).

In the present study, the ROS level was increased when UCP2 was knocked down under experimental septic conditions. This is consistent with the result observed for MMP, and could explain the protective role of UCP2 on a different level.

Cellular ATP, which is the special energy currency in the cell, is also an important indicator of mitochondrial function as it is generated from the coupling of electron transport and proton dynamics through the electron transport chain (ETC). UCP2 decouples oxidation and phosphorylation in mitochondria by reducing the proton concentration difference on both sides of the mitochondrial inner membrane and ATP synthesis. Our present study found that sepsis damaged astrocytes and led to low levels of ATP, while UCP2 silencing rescued the ATP levels. This finding of the function of UCP2 was previously identified.

Our experimental results showed that mitochondrial dysfunction aggravated oxidative stress, leading to a decrease in the MMP and ATP levels under septic conditions. Knockdown of UCP2 aggravated the destruction of mitochondrial ultrastructure, caused more severe edema and damage to mitochondrial inner membrane, and increased MMP disruption, suggesting that UCP2 may play a positive role under SAE conditions. However, the neuroprotective effect of UCP2 in sepsis-associated encephalopathy remains to be determined. The role of UCP2 in neurons in the brain requires further study.

In conclusion, our study showed that co-stimulation with LPS+IFN- $\gamma$ triggered mitochondrial disorder in astrocytes and that knockdown of UCP2 by adenoviral transfection exacerbated mitochondrial injury, indicating that UCP2 has a positive effect in astrocytes under septic conditions. 


\section{Acknowledgements}

Not applicable.

\section{Funding}

This work was supported by the National Natural Science Foundation of China $(81272070,81601664)$ and the Guangzhou Key Laboratory of Inflammatory and Immune Diseases [(2012)224-226].

\section{Availability of data and materials}

The datasets used during the present study are available from the corresponding author upon reasonable request.

\section{Authors' contributions}

QZ and WP conceived and designed this study. WP, JH, YZ, YD, SL and JZ performed the experiments and analyzed the data. QZ, and WP wrote, edited and reviewed the manuscript. $\mathrm{JL}$ was responsible for the planning of experimental scheme and the arrangement of experimental tasks. WP and $\mathrm{JH}$ contributed equally to this work. All authors read and approved the final submitted version of the manuscript and agree to be accountable for all aspects of the research in ensuring that the accuracy or integrity of any part of the work are appropriately investigated and resolved.

\section{Ethics approval and consent to participate}

This study was approval by the Nanfang Hospital Ethics Committee (NFYY-2017-39). The animals used in the study were provided by Southern Medical University Animal Experiment Center [SCXK(Guangdong)2016-0041], and passed the experimental animal quality inspection in Guangdong Province (no. 44002100020527).

\section{Patient consent for publication}

Not applicable.

\section{Competing interests}

The authors declare that there have no competing interests.

\section{References}

1. Hawiger J, Veach RA and Zienkiewicz J: New paradigms in sepsis: From prevention to protection of failing microcirculation. J Thromb Haemost 13: 1743-1756, 2015.

2. Chaudhry N and Duggal AK: Sepsis associated encephalopathy. Adv Med 2014: 762320, 2014.

3. Helbing DL, Bohm L and Witte OW: Sepsis-associated encephalopathy. CMAJ 190: E1083, 2018.

4. Eyenga P, Roussel D, Morel J, Rey B, Romestaing C, GueguenChaignon V,Sheu SS and Viale JP:Time course of liver mitochondrial function and intrinsic changes in oxidative phosphorylation in a rat model of sepsis. Intensive Care Med Exp 6: 31, 2018.

5. Dutra MR, Feliciano RD, Jacinto KR, Gouveia TL, Brigidio E, Serra AJ, Morris M, Naffah-Mazzacoratti MD and Silva JA Jr: Protective role of UCP2 in oxidative stress and apoptosis during the silent phase of an experimental model of epilepsy induced by pilocarpine. Oxid Med Cell Longev 6: 6736721, 2018.
6. Chen XL, Wang Y, Peng WW, Zheng YJ, Zhang TN, Wang PJ, Huang JD and Zeng QY: Effects of interleukin-6 and IL-6/AMPK signaling pathway on mitochondrial biogenesis and astrocytes viability under experimental septic condition. Int Immunopharmacol 59: 287-294, 2018.

7. Molofsky AV and Deneen B: Astrocyte development: A guide for the perplexed. Glia 63: 1320-1329, 2015.

8. Donadelli M, Dando I, Fiorini C and Palmieri M: UCP2, a mitochondrial protein regulated at multiple levels. Cell Mol Life Sci 71: 1171-1190, 2014.

9. Moukdar F, Robidoux J, Lyght O, Pi J, Daniel KW and Collins S: Reduced antioxidant capacity and diet-induced atherosclerosis in uncoupling protein-2-deficient mice. J Lipid Res 50: 59-70, 2009.

10. Haines B and Li PA: Overexpression of mitochondrial uncoupling protein 2 inhibits inflammatory cytokines and activates cell survival factors after cerebral ischemia. PLoS One 7: e31739, 2012.

11. Vogler S, Pahnke J, Rousset S, Ricquier D, Moch H, Miroux B and Ibrahim SM: Uncoupling protein 2 has protective function during experimental autoimmune encephalomyelitis. Am J Pathol 168: 1570-1575, 2006.

12. Chen W, Luo S, Xie P, Hou T, Yu T and Fu X: Overexpressed UCP2 regulates mitochondrial flashes and reverses lipopolysaccharide-induced cardiomyocytes injury. Am J Transl Res 10: 1347-1356, 2018.

13. Singer M: The role of mitochondrial dysfunction in sepsis-induced multi-organ failure. Virulence 5: 66-72, 2014.

14. Deng S, Yang Y, Han Y, Li X, Wang X, Li X, Zhang Z and Wang Y: UCP2 inhibits ROS-mediated apoptosis in A549 under hypoxic conditions. PLoS One 7: e30714, 2012.

15. Zhao YZ, Gao ZY, Ma LQ, Zhuang YY and Guan FL: Research on biogenesis of mitochondria in astrocytes in sepsis-associated encephalopathy models. Eur Rev Med Pharmacol Sci 21: 3924-3934, 2017.

16. Lu M, Zhao FF, Tang JJ, Su CJ, Fan Y, Ding JH, Bian JS and Hu G: The neuroprotection of hydrogen sulfide against MPTP-induced dopaminergic neuron degeneration involves uncoupling protein 2 rather than ATP-sensitive potassium channels. Antioxid Redox Signal 17: 849-859, 2012.

17. Wang T, Chen Y, Zhang J, Chen G, Zhang J, Huang J and Zeng Q: Protective effects and mechanism of insulin on brain in septic rats. Chin J Appl Clin Pediatr 32: 856-860, 2017.

18. Livak KJ and Schmittgen TD: Analysis of relative gene expression data using real-time quantitative PCR and the 2(-Delta Delta C(T)) method. Methods 25: 402-408, 2001.

19. Lyu J, Zheng G, Chen Z, Wang B, Tao S, Xiang D, Xie M, Huang J, Liu C and Zeng Q: Sepsis-induced brain mitochondrial dysfunction is associated with altered mitochondrial Src and PTP1B levels. Brain Res 1620: 130-138, 2015.

20. Chen GD, Zhang JL, Chen YT, Zhang JX, Wang T and Zeng QY: Insulin alleviates mitochondrial oxidative stress involving upregulation of superoxide dismutase 2 and uncoupling protein 2 in septic acute kidney injury. Exp Ther Med 15: 3967-3975, 2018.

21. Zheng G, Lyu J, Liu S, Huang J, Liu C, Xiang D, Xie M and Zeng Q: Silencing of uncoupling protein 2 by small interfering RNA aggravates mitochondrial dysfunction in cardiomyocytes under septic conditions. Int J Mol Med 35: 1525-1536, 2015.

22. Lu M, Sun XL, Qiao C, Liu Y, Ding JH and Hu G: Uncoupling protein 2 deficiency aggravates astrocytic endoplasmic reticulum stress and nod-like receptor protein 3 inflammasome activation. Neurobiol Aging 35: 421-430, 2014.

23. Normoyle KP, Kim M, Farahvar A, Llano D, Jackson K and Wang $\mathrm{H}$ : The emerging neuroprotective role of mitochondrial uncoupling protein-2 in traumatic brain injury. Transl Neurosci 6: 179-186, 2015.

24. Braun RJ: Mitochondrion-mediated cell death: Dissecting yeast apoptosis for a better understanding of neurodegeneration. Front Oncol 2: 182, 2012.

25. Xiong W, Hua J, Liu Z, Cai W, Bai Y, Zhan Q, Lai W, Zeng Q, Ren H and Xu D: PTEN induced putative kinase 1 (PINK1) alleviates angiotensin II-induced cardiac injury by ameliorating mitochondrial dysfunction. Int J Cardiol 266: 198-205, 2018.

This work is licensed under a Creative Commons Attribution-NonCommercial-NoDerivatives 4.0 International (CC BY-NC-ND 4.0) License. 